\title{
Susceptibility Pattern of Bacterial Isolates from Catheterised Patients in a Referral Hospital
}

\author{
Tongen Roel ${ }^{1}$, Biswajeet Sahoo $^{2}$, Kh. Sulochana Devi ${ }^{3}$, L. Deban Singh ${ }^{4}$, \\ Yompe Kamki ${ }^{5}$, Aribam Vijaya Devi ${ }^{6}$ \\ IMD ( Microbiology), Medical Officer, District Hospital, Chandel \\ 2 Post Graduate student Dept. Of Microbiology, Regional Institute of Medical Sciences, Imphal. \\ 3 Professor and Head Dept. Of Microbiology, Regional Institute of Medical Sciences, Imphal. \\ 4 Professor Dept. Of Anesthesiology, Regional Institute of Medical Sciences, Imphal \\ 5MD ( Microbiology), Medical Officer, CHC Likabali, Arunachal Pradesh. \\ 6Senior Resident Dept. Of Microbiology, Regional Institute of Medical Sciences, Imphal
}

\begin{abstract}
:
Introduction: Catheter associated urinary tract infection is one of the most important Device associated infection (DAI) and it is also one of the leading causes of mortality and morbidity in hospitalized patients.

Aims And Objects: To isolate and identify organisms causing catheter associated urinary tract infection and determine the antibiotic susceptibility pattern of the isolates.

Materials And Methods: This study was carried out from November 2011-October 2013, a total of 71 samples were collected. These samples were processed using routine biochemical test. Antibiotic susceptibility was determined by Kirby-Bauer disc diffusion method as per CLSI recommendations.

Result: A total of 11 samples were positive and 12 organisms were isolated, of which 3 were Gram positive and 9 were Gram negative organisms. The most common Gram positive isolate was S.aureus and E.coli was the most common Gram negative isolate. All the S.aureus isolates were Methicillin resistant (MRSA). But it was $100 \%$ sensitivity to vancomycin, linezolid and co-trimoxazole. As for the gram negative organisms, it was most sensitive to imipenem.

Conclusion: Gram negative isolates were the most common pathogens in catheterised patients. Measures to control DAI are needed to decrease morbity and mortality among the admitted patient, for the hospital to develop a strict antibiotic policy and also the information is needed for creation of such devices which can prevent device associated infections.
\end{abstract}

\section{Introduction}

Catheter associated urinary tract infection is one of the most important Device associated infection (DAI) and it is one of the leading causes of morbidity and mortality in hospitalized patients. The frequency of such infection particularly in hospitalised patients and the agents and their resistance rates should be identified for better treatment, infection control and creation of a better hospital antibiotic policy. Studies examining nosocomial infections for the purpose of assessing the nosocomial infection rate are one of the most important indicators of the quality of health services. ${ }^{[1]}$ A person is said to be suffering from catheter associated UTI if he developed one or more of the following conditions i.e fever [temp $\geq 38^{\circ} \mathrm{C}$ ] without any other known cause, increase in urgency or suprapubic tenderness with urine culture showing more than $10^{5} \mathrm{CFU} / \mathrm{ml}$ of

urine with not more than 2 types of organisms, if urine shows pyuria with more than 3 WBC seen on Gram stain and strong clinical suspicion. ${ }^{[2]}$

Catheter-associated UTI (CAUTI) is the most common nosocomial infection, accounting for $>1$ million cases in hospitals and nursing homes. The risk of UTI increases with increasing duration of catheterization, material of the catheter and other comorbid and other instrumentation. The UTIs from catheterized and hospitalized patients are known to include strains which are resistant to antimicrobials ${ }^{[2]}$. The infection control measures in Indian hospitals are, however, constrained by the paucity of research in the field and the lack of hospital-specific data on nosocomial infections. Baseline estimates of the magnitude of the problem and the extent of antimicrobial resistance among the nosocomial pathogens are the minimum essential prerequisites for any hospital infection control programme. ${ }^{[3]}$ 


\section{Materials And Methods}

It is a Cross sectional study and it include patients without urinary tract infection at the time of admission in the hospital and those patients who developed infection within 48 hours of admission were excluded from the study. A total of 71 urine samples were collected from the urinary catheter sampling port with a sterile needle and syringe. Urine samples were processed within 1-2 hours of collection or kept at $4^{\circ} \mathrm{C}$ when delay is unavoidable. The urine samples was inoculated on MacConkey agar, Blood agar and CLED (Cystine lactose electrolyte deficient agar) using a standard loop and incubated aerobically at

$37^{\circ} \mathrm{C}$ overnight. A sample showing growth of more than $10^{5} / \mathrm{ml}$ was recorded as significant bacteriuria. The colonies morphology was studied and recorded. The colonies were then subjected to a series of tests for the identification of the isolates. Antibiotic susceptibility testing was performed by Kirby Bauer disc diffusion method as per CLSI recommendations. ${ }^{[79]}$ The medium used was Mueller Hinton Agar (MHA).

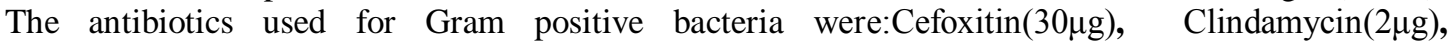

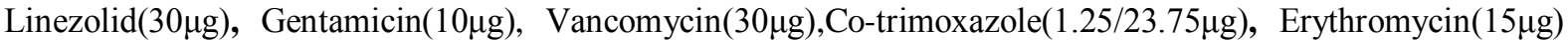

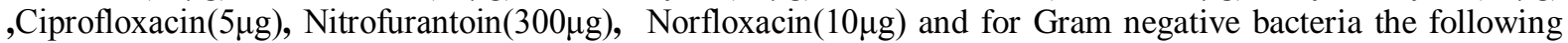

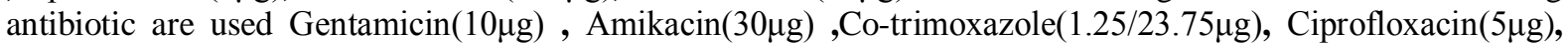
Nitrofurantoin $(300 \mu \mathrm{g}), \quad$ Norfloxacin $(10 \mu \mathrm{g}), \quad$ Ceftazidime $(30 \mu \mathrm{g}), \quad$ Imipenem $(10 \mu \mathrm{g})$, Piperacillin+Tazobactum $(100 \mu \mathrm{g} / 10 \mu \mathrm{g})$, Ceftriaxone $(30 \mu \mathrm{g})$ and Colistin $(10 \mu \mathrm{g})$

\section{Results And Observation}

A total of 71 urine samples were collected during a period of two years from the admitted patients . The data collected were then analysed. Out of the 71 samples, $11(15.49 \%)$ were culture positive, all of them showed monomicrobial growth except for one sample which showed polymicrobiol growth. A total of 12 organisms were isolated and Gram negative organisms were the most common pathogen. The most common Gram negative isolates was E.coli followed by Klebsiella pneumonia, Pseudomonas aeruginosa, Enterobacter cloacae and Acinetobacter baumannii andhe Gram positive isolate was S.aureus as showed in table 1.

Table 1: Bacterial profile of urinary isolates from catheterised patients

\begin{tabular}{|l|l|l|l|l|}
\hline Sl. no. & Antibiotic & Clinical disease & Organism isolated & Colony count \\
\hline 1. & ceftriaxone & CCF & Staph.aureus & $>10^{5} \mathrm{CFU} / \mathrm{ml}$ \\
\hline 2. & NA & IHD with VD & Staph.aureus & $>10^{5} \mathrm{CFU} / \mathrm{ml}$ \\
\hline 3. & NA & CCF & Staph.aureus & $>10^{5} \mathrm{CFU} / \mathrm{ml}$ \\
\hline 4. & $\begin{array}{l}\text { Ceftazidime, } \\
\text { Cotrimoxzaxole }\end{array}$ & $\begin{array}{l}\text { C3 cervical spine } \\
\text { fracture }\end{array}$ & Enterobacter cloacae & $>10^{5} \mathrm{CFU} / \mathrm{ml}$ \\
\hline 5. & Linezolid & CVA & Klebsiella spp. & $>10^{5} \mathrm{CFU} / \mathrm{ml}$ \\
\hline 6. & Piperacillin + tazobactum & Encephalitis & Acinetobacter baumannii & $>10^{5} \mathrm{CFU} / \mathrm{ml}$ \\
\hline 7. & Piperacillin + tazobactum & Pontine haemorrhage & Klebsiella pneumoniae & $>10^{5} \mathrm{CFU} / \mathrm{ml}$ \\
\hline 8. & NA & CVA & E.coli & $>10^{5} \mathrm{CFU} / \mathrm{ml}$ \\
\hline 9. & Ceftriaxone + sulbactum & Quadriparesis & Pseudomonas aeruginosa & $>10^{5} \mathrm{CFU} / \mathrm{ml}$ \\
\hline 10. & NA & CVA & E.coli & $>10^{5} \mathrm{CFU} / \mathrm{ml}$ \\
\hline 11. & NA & CVA & E.coli & $>10^{5} \mathrm{CFU} / \mathrm{ml}$ \\
\hline 12. & NA & CVA & Pseudomonas aeruginosa & $>10^{5} \mathrm{CFU} / \mathrm{ml}$ \\
\hline
\end{tabular}

$* \mathrm{NA}=$ Not on antibiotics, $\mathrm{CCF}=$ Congestive cardiac failure, IHD = Ischaemic heart disease, $\mathrm{VD}=$ Ventricular dysfunction, CVA = Cerebrovascular accident

Out of the 71 patients, 41 had already received antibiotics, while the 30 patients were not on antibiotics. Among those receiving antibiotics culture was positive in 6 of the samples, and in those patients who were not on antibiotics, 5 of the samples were culture positive, 10 of the samples showed monomicrobial growth while the remaining 1 sample(not on antibiotic) showed polymicrobial growth of E.coli and Pseudomonas aeruginosa. The Gram negative isolates were most sensitive to imipenem and least sensitive to ceftriaxone. As for the Gram positive isolates it was $100 \%$ sensitive to co-trimoxazole, vancomycin and linezolid but it was $100 \%$ resistant to cefoxitin (MRSA), as showed in the table 2 and 3 below.

Table 2: Antibiotic sensitivity pattern of Gram negative isolates from urine

\begin{tabular}{|l|l|l|l|l|l|}
\hline Antibiotic & $\begin{array}{l}\text { Pseudomonas } \\
\text { aeruginosa } \\
(\mathrm{n}=2)\end{array}$ & $\begin{array}{l}\text { E.coli } \\
(\mathrm{n}=3)\end{array}$ & $\begin{array}{l}\text { Klebsiella } \\
\mathrm{spp} . \\
(\mathrm{n}=2)\end{array}$ & $\begin{array}{l}\text { Acinetobacter } \\
\text { baumannii } \\
(\mathrm{n}=1)\end{array}$ & $\begin{array}{l}\text { Enterobacter } \\
\text { cloacae}(\mathrm{n}=1)\end{array}$ \\
\hline Ciprofloxacin & $1(50 \%)$ & $1(33.33 \%)$ & $1(50 \%)$ & 0 & 0 \\
\hline Norfloxacin & $1(50 \%)$ & $1(33.33 \%)$ & 0 & 0 & $1(100 \%)$ \\
\hline Nitrofurantoin & 0 & $2(66.67 \%)$ & 0 & 0 & 0 \\
\hline Co-trimoxazole & NA & 0 & $1(50 \%)$ & $1(100 \%)$ & 0 \\
\hline Amikacin & $1(50 \%)$ & $3(100 \%)$ & $1(50 \%)$ & 0 & $1(100 \%)$ \\
\hline Gentamicin & $2(100 \%)$ & $3(100 \%)$ & $1(50 \%)$ & 0 & 0 \\
\hline
\end{tabular}


Susceptibility pattern of bacterial isolates from catheterised patients in a referral hospital

\begin{tabular}{|l|l|l|l|l|l|}
\hline Ceftazidime & $1(50 \%)$ & 0 & 0 & 0 & 0 \\
\hline Ceftriaxone & 0 & 0 & 0 & 0 & 0 \\
\hline Imipenem & $1(50 \%)$ & $3(100 \%)$ & $2(100 \%)$ & $1(100 \%)$ & $1(100 \%)$ \\
\hline $\begin{array}{l}\text { Piperacillin } \\
\text { Tazobactum }\end{array}$ & $1(50 \%)$ & 0 & & 0 & 0 \\
\hline Colistin & $1(50 \%)$ & NA & NA & NA & NA \\
\hline
\end{tabular}

*NA $=$ Not applicable

Table 3: Antibiotic sensitivity pattern of Gram positive isolates from urine

\begin{tabular}{|l|l|}
\hline Antibiotic & Staph.aureus $(\mathrm{n}=3)$ \\
\hline Ciprofloxacin & $1(33.33 \%)$ \\
\hline Co-trimoxazole & $3(100 \%)$ \\
\hline Cefoxitin & 0 \\
\hline Gentamicin & $2(66.67 \%)$ \\
\hline Erythromycin & 0 \\
\hline Vancomycin & $3(100 \%)$ \\
\hline Clindamycin & $2(66.67 \%)$ \\
\hline Linezolid & $3(100 \%)$ \\
\hline Norfloxacin & 0 \\
\hline Nitrofurantoin & $1(33.33 \%)$ \\
\hline
\end{tabular}

Table 4: Relationship of duration of device with the number of positive

\begin{tabular}{|l|l|l|l|}
\hline Duration of device in days & No. of negative sample & No. of positive sample & Total \\
\hline $2-7$ & 45 & 5 & 50 \\
\hline$>8$ & 15 & 6 & 21 \\
\hline Total & 06 & 11 & 71 \\
\hline
\end{tabular}

$\mathrm{X}^{2}=3.89 \quad \mathrm{P}$ value $=0.048$

From Table 4 we can see that the number of bacteria isolated increases with the duration of use of the device and it was found to be significant. And the DAI rate was found to be 23.80/1000 device days.

\section{Discussion}

Device associated infection is associated with high morbidity, mortality and have a considerable impact on health costs which is usually due to antimicrobial resistant bacteria. The bacterial profile and antimicrobial susceptibility pattern are constantly evolving. So, study of the bacterial profile with antibiotic susceptibility pattern plays an important role in effective management of Device-associated Infections (DAIs), formulation of hospital antibiotic policy for DAIs, and also for the creation of devices which will have very less rate of infections. Many studies have been undertaken to determine the organisms responsible for DAIs but results have varied in different countries, which might be due to many factors, such as hand hygiene, administration of different antibiotics etc. Out of the 71 urine samples, 11(15.49\%)

of the samples were positive by culture and the DAI rate for catheterized patients was 23. 80/1000 device days. All of the samples showed monomicrobiol growth except 1 sample which showed polymicrobiol growth. It is similar to the finding of Melaku S et al ${ }^{[4]}$ where out of the 118 urine samples only one sample showed polymicrobiol growth and E.coli and Staphylococcus aureus were the most common isolates, which is a similar finding in this study. Djordjevic $\mathrm{Z}$ et a ${ }^{[5]}$ found that hospitalisation in an ICU bears high risk of hospital infections (HIs), especially of urinary tract infections caused by Gram negative bacteria, in patients with longer hospital stay or co-morbidities, and in those who have had surgical interventions or prolonged use of a urinary bladder catheter. Urinary bladder catheterization longer than seven days and longer hospital stay was associated with DAIs which is a similar finding in the present study.Gram negative organisms predominate in hospital acquired urinary tract infections, almost all of which are associated with urethral catheterization. Recent U. S. data indicate that $\mathrm{E}$. coli is the most common etiologic Gram negative organism, followed in descending order of frequency by Pseudomonas aeruginosa, Klebsiella species, Enterobacter species, and Acinetobacter baumannii and they have also found that most of the ESBL producing E.coli were also resistant to fluoroquinolones.

In the present study E.coli was the most common organism among the Gram negative isolates followed by Pseudomonas aeruginosa.Laupland $\mathrm{KB}^{[6]}$ observed that most of the UTIs were monomicrobial infections but in 19 cases two organisms were identified. Antibiotic resistant organisms were identified in 14\% (53/375) of isolates. These organisms were Escherichia coli in 29 (55\%), Pseudomonas aeruginosa in 12 (23\%), Klebsiella species in $5(9 \%)$, and other Gram negative enterics in 7 (13\%). None of the Staphylococcus aureus were methicillin resistant. Imipenem resistance was identified in one of seven isolates of Pseudomonas aeruginosa tested, whereas in the present study all the 3 Staphylococcus aureus were MRSA and out of 2 Pseudomonas aeruginosa 1 was resistant to imipenem. 
Antimicrobial treatment suppressed the endogenous bacterial flora of the urinary system, and leads to CA-UTI. In the cohort study by Xie $\mathrm{D}^{[7]} 65.67 \%$ (44/67) of CAUTI yielded positive results by bacteriologic tests. There were 47 pathogenic bacteria isolated. Of these isolates, fungi were the most frequently isolated $(21.28 \%$,

10/47), followed by Escherichia coli (17.02\%, 8/47), and Pseudomonas aeruginosa (10.64\%, 5/47). In their study Escherichia coli resistance for ciprofloxacin was $88 \%$, and Pseudomonas aeruginosa showed absolute resistance to ciprofloxacin, amikacin, ceftazidime and meropenem. In the present study urinary isolates of Escherichia coli resistance for ciprofloxacin was $66.67 \%$ and sensitivity to amikacin and imipenem were $100 \%$. The Escherichia coli was $100 \%$ resistant to Ceftazidime. The Pseudomonas aeruginosa isolates were $50 \%$ sensitive to amikacin, ciprofloxacin, ceftazidime and imipenem and Gram negative bacterias were the most common isolates comprising $75 \%$ of the urinary isolates. Due to increased and indiscriminate use of antibiotics, there has been emergence of antibiotic resistance among microorganisms. This also hold equally good for DAIs pathogens. This phenomenon of drug resistance differ from one place to another. Therefore, the purpose of this study was to assess the DAIs rate and the degree of antimicrobial susceptibility pattern among the key pathogen causing DAIs to the commonly used antibiotics in the hospital. The present study observed that vancomycin and linezolid appeared to maintain activity against Gram positive isolates in $100 \%$ of the cases. Whereas Dhand $\mathrm{A}^{[8]}$ found that only $99 \%$ of the Staphylococcus isolates were sensitive to vancomycin.

\section{Conclusion}

In this study the rate of device-associated infection was 23.80/1000 device days and most of the isolates from the urine were Gram negative bacteria 9(75\%), all of them were resistant to ceftriaxone. There was high resistant to piperacillin-tazobactum, only 1 isolates was sensitive to it, but it was highly sensitive to imipenem only 1 of the isolates was resistant to it. Three Staphylococcus aureus were isolated from urine, all of them were cefoxitin resistant (MRSA) and it was $100 \%$ resistant to erythromycin and norfloxacin but it was $100 \%$ sensitive to vancomycin, linezolid and co-trimoxazole.In order to reduce device-associated nosocomial infections in the ICU following suggestion should be considered:

Monitoring of duration of device and removal of it as soon as possible.

$>$ Regular surveillance.

$>$ Antibiotic susceptibility testing prior to antibiotic therapy.

$>$ There should be coordinate participation of the Microbiologist, Clinician, Nursing personal and Hospital infection control team.

Measures to control DAI are thus needed to decrease morbity and mortality among the admitted patient, formulation of use of duration of catheter which is a major factor for DAIs, for the hospital to develop a strict antibiotic policy for DAIs and also the information would be useful for creation of such devices which can prevent device associated infections.

\section{References}

[1]. Dogru A, Sargin F, Celik M, sagiroglu A E, Goksel M M, Sayhan H. The rate of Device-Associated nosocomial Infections in a Medical Surgical Intensive Care Unit of a Traning and Research Hospital in Turkey: One year Outcomes. Jpn. J. of Infect. Dis. 2010; 63:95-8.

[2]. Singh S, Pandya Y, Patel R, Wilson A, Trivedi S. Surveillance of device-associated infections at a teaching hospital in rural Gujarat-India. Indian J of Med Microbiol. 2010; 28(4): 342-7.

[3]. Kamat U S, Fereirra A, Amonkar D, Motghare D D, Kulkarni M S. Epidemiology of hospital acquired urinary tract infections in a medical college hospital in Goa. Indian J Urol. 2009 Jan-Mar; 25(1): 76-80.Clinical and Laboratory Standards Institute (CLSI). Perfomance standards for antimicrobial susceptibility testing, $19^{\text {th }}$ informational supplement, 2009 Jan.;29(3):M07-A8.

[4]. Melaku S, Kibret M, Abera B, Gebre-Sellassie S. Antibiogram of nosocomial urinary tract infections in Felege Hiwot referral hospital, Ethiopia. Afr Health Sci. 2012 June; 12(2): 134-9.

[5]. Djordjevic Z, Jankovic S, Gajovic O, Djonovic N, Folic N, Bukumiric Z. Hospital infections in a neurological intensive care unit: incidence, causative agents and risk factors .J Infect Dev Ctries. 2012; 6(11):798-805.

[6]. Laupland K B, Bagshaw S M, Gregson D B, Kirkpatrick A W, Ross T, Church D L. Intensive care unit-acquired urinary tract infections in a regional critical care system. Crit Care. 2005; 9(2):R60-R65.

[7]. Xie D, Lai R, Nie S. Surveys of catheter-associated urinary tract infection in a university hospital intensive care unit in China. Braz J Infect Dis.2011May/June;15(3). Available on http://dx.doi.org/10.1590/S14138670201100030001. Accessed on September, 21,2013 .

[8]. Dhand A, Sakoulas G. Reduced vancomycin susceptibility among clinical Staphylococcus aureus isolates ('the MIC Creep'): implications for therapy. Med

[9]. Reports 2012; 4(4). Available on http://f1000.com/reports/m/4/4. Accessed on september,15,2013. 